\title{
On the taxonomic status of the intracellular bacterium Wolbachia pipientis: should this species name include the intracellular bacteria of filarial nematodes?
}

The recent article by Lo et al. (2007) aiming to recognize the Wolbachia endobacteria of arthropods and filarial nematodes as Wolbachia pipientis, while admirable for attempting to codify the nomenclature in this field of research, may not address the stark differences of the endobacteria found in nematodes compared with the endobacteria found in arthropods.

Lo et al. (2007) propose that all endobacteria of arthropods and nematodes commonly called Wolbachia be formally declared as Wolbachia pipientis. Part of their reasoning is based on the difficulty of assigning the strains to monophyletic species due to the lack of an appropriate outgroup and the fact that to name arthropod strains after the species they infect would be unwieldy because of the huge number of arthropod species infected with these endobacteria (Lo et al., 2007). This was the consensus reached at the 2004 international conference on $W$. pipientis (Heron Island, Australia, August 2004). However, in 2005, the genome of Wolbachia from the filarial nematode Brugia malayi $(w \mathrm{Bm})$ was published (Foster et al., 2005) and compared with the previously published genome of the Wolbachia from Drosophila melanogaster (wMel) (Wu et al., 2004). Analysis of the genome and the biology of the Wolbachia in nematodes argues against including these endobacteria with those from arthropods as W. pipientis.

The $w \mathrm{Bm}$ genome is $\sim 200 \mathrm{~kb}$ smaller than that of $w \mathrm{Mel}$, resulting in fewer predicted proteins (Foster et al., 2005). In addition to the difficulty in making phylogenetic

The names used in this manuscript are used within the context of general discussion as covered by Rule 28a of the 1990 revision of the Bacteriological Code and are not being formally proposed. As such, they do not meet any of the criteria required for valid publication of a name. trees without an appropriate outgroup, there is a high degree of recombination in arthropod strains, characteristic of horizontally transmitted bacteria, requiring multilocus strain typing for reliable identification (Baldo et al., 2006). However, while $w \mathrm{Mel}$ has the genes necessary for recombination, prophage sequences that could facilitate recombination and promote exchange of genes or gene fragments and a high proportion of repetitive DNA elements (14.2\%) (Wu et al., 2004), wBm has no active system for DNA recombination and no prophages and a much smaller proportion of repetitive elements $(5.4 \%)$. As a result, $w \mathrm{Bm}$ shows no or little recombination (Jiggins, 2002). Despite the difficulties in making phylogenetic trees for Wolbachia, all trees consistently group the varied nematode Wolbachia together into supergroups C, D and F (Casiraghi et al., 2005), with the phylogeny of the endobacteria in supergroups C and D being concordant with the phylogeny of their hosts (Casiraghi et al., 2001, 2004; Fenn et al., 2006).

In addition to molecular evidence for nematode Wolbachia being different from the endobacteria in arthropods, evidence can be found by looking at the biology of these organisms. The first is that all adult worms in species infected with Wolbachia have the endobacteria. Besides a block in embryonic and larval development, adult worms die when the endobacteria are depleted with antibiotics (Taylor et al., 2005), i.e. they are mutualists rather than parasites. In contrast, not all members of an arthropod population are infected with Wolbachia, and the majority of these infections can be cured with antibiotics without adverse effects on the host. Second, horizontal transfer of Wolbachia in arthropods has been described in nature (Turelli \& Hoffmann, 1991; Vavre et al., 1999), and stable infections of the endobacteria from one species to another have been performed in the lab using micro-injection or natural mechanisms (Heath et al., 1999; Riegler et al., 2004). In contrast, micro-injection of Wolbachia from the nematode Litomosoides sigmodontis into the Wolbachia-free nematode Acanthocheilonema viteae resulted in apparent replication of the endobacteria, but none were detected in the larvae released from these injected worms (Hartmann et al., 2003).

Unfortunately, the endobacteria were not localized in the worms to see where they had established themselves - in cells or only in the body cavity. Attempts to infect Caenorhabditis elegans or insect cell lines with nematode Wolbachia that are then able to replicate have been unsuccessful (B. Slatko, unpublished results). This probably reflects the fact that the Wolbachia in nematodes are mutualists, and their hosts have become dependent on their endosymbionts for embryonic and larval development as well as adult survival (Pfarr \& Hoerauf, 2006).

Furthermore, the significant phylogenetic distance of the hosts should also be considered. Arthropods and nematodes are in different animal phyla and are estimated to be separated by nearly a billion years of evolution (Wang et al., 1999).

In summary, we have attempted to define several points that argue for a different species name for the Wolbachia in nematodes. Until the endobacteria in the genus Wolbachia are formally characterized, typed and named according to the Bacteriological Code (Lapage et al., 1992), we believe that there is enough evidence to support naming, for purposes of discussion, the Wolbachia of supergroups C and D after a key host species in each supergroup, e.g. 'Wolbachia volvulus' (Onchocerca volvulus) and 'Wolbachia malayi' (Brugia malayi), 
respectively. The endobacteria of Mansonella spp., in Wolbachia supergroup

$\mathrm{F}$, which have both nematode and arthropod hosts, should remain $W$. pipientis until more study clarifies their phylogeny. By assigning different names to Wolbachia of the C and D supergroups, their unique biology and phylogeny will be better highlighted and the taxonomy of Wolbachia will be more in line with the biology of these fascinating bacteria.

\section{Kenneth Pfarr ${ }^{1}$, Jeremy Foster ${ }^{2}$, Barton Slatko ${ }^{2}$, Achim Hoerauf ${ }^{1}$ and Jonathan A. Eisen ${ }^{3}$}

${ }^{1}$ Institute for Medical, Microbiology, Immunology and Parasitology, University Clinic Bonn, Sigmund-Freud-Str. 25, D-53105 Bonn, Germany

${ }^{2}$ Molecular Parasitology Division, New England Biolabs, 240 County Road, Ipswich, MA 01938, USA

${ }^{3}$ UC Davis Genome Center, University of California, Davis, CA 95616, USA

Correspondence: Kenneth M. Pfarr (pfarr@parasit.meb.uni-bonn.de)

Baldo, L., Dunning Hotopp, J. C., Jolley, K. A., Bordenstein, S. R., Biber, S. A., Choudhury, R. R., Hayashi, C., Maiden, M. C., Tettelin, H. \& Werren, J. H. (2006). Multilocus sequence typing system for the endosymbiont Wolbachia pipientis. Appl Environ Microbiol 72, 7098-7110.

Casiraghi, M., Favia, G., Cancrini, G., Bartoloni, A. \& Bandi, C. (2001). Molecular identification of Wolbachia from the filarial nematode
Mansonella ozzardi. Parasitol Res 87, 417-420.

Casiraghi, M., Bain, O., Guerrero, R., Martin, C., Pocacqua, V., Gardner, S. L., Franceschi, A. \& Bandi, C. (2004). Mapping the presence of Wolbachia pipientis on the phylogeny of filarial nematodes: evidence for symbiont loss during evolution. Int J Parasitol 34, 191-203.

Casiraghi, M., Bordenstein, S. R., Baldo, L., Lo, N., Beninati, T., Wernegreen, J. J., Werren, J. H. \& Bandi, C. (2005). Phylogeny of Wolbachia pipientis based on gltA, groEL and ftsZ gene sequences: clustering of arthropod and nematode symbionts in the F supergroup, and evidence for further diversity in the Wolbachia tree. Microbiology 151, 4015-4022.

Fenn, K., Conlon, C., Jones, M., Quail, M. A., Holroyd, N. E., Parkhill, J. \& Blaxter, M. (2006). Phylogenetic relationships of the Wolbachia of nematodes and arthropods. PLoS Pathog 2, e94.

Foster, J., Ganatra, M., Kamal, I., Ware, J., Makarova, K., Ivanova, N., Bhattacharyya, A., Kapatral, V., Kumar, S. \& other authors (2005). The Wolbachia genome of Brugia malayi: endosymbiont evolution within a human pathogenic nematode. PLoS Biol 3, el21.

Hartmann, N., Stuckas, H., Lucius, R., Bleiß, W., Theuring, F. \& Kalinna, B. H. (2003). Transspecies transfer of Wolbachia: microinjection of Wolbachia from Litomosoides sigmodontis into Acanthocheilonema viteae. Parasitology 126, 503511.

Heath, B. D., Butcher, R. D., Whitfield, W. G. \& Hubbard, S. F. (1999). Horizontal transfer of Wolbachia between phylogenetically distant insect species by a naturally occurring mechanism. Curr Biol 9, 313-316.

Jiggins, F. M. (2002). The rate of recombination in Wolbachia bacteria. Mol Biol Evol 19, 1640-1643.

Lapage, S. P., Sneath, P. H. A., Lessel, E. F., Skerman, V. B. D., Seeliger, H. P. R. \& Clark, W. A. (1992). International Code of Nomenclature of Bacteria (1990 Revision). Bacteriological Code.
Washington, DC: American Society for Microbiology.

Lo, N., Paraskevopoulos, C., Bourtzis, K O'Neill, S. L., Werren, J. H., Bordenstein, S. R. \& Bandi, C. (2007). Taxonomic status of the intracellular bacterium Wolbachia pipientis. Int $J$ Syst Evol Microbiol 57, 654-657.

Pfarr, K. M. \& Hoerauf, A. M. (2006). Antibiotics which target the Wolbachia endosymbionts of filarial parasites: a new strategy for control of filariasis and amelioration of pathology. Mini Rev Med Chem 6, 203-210.

Riegler, M., Charlat, S., Stauffer, C. \& Mercot, H. (2004). Wolbachia transfer from Rhagoletis cerasi to Drosophila simulans: investigating the outcomes of host-symbiont coevolution. Appl Environ Microbiol 70, 273-279.

Taylor, M. J., Makunde, W. H., McGarry, H. F., Turner, J. D., Mand, S. \& Hoerauf, A. (2005). Macrofilaricidal activity after doxycycline treatment of Wuchereria bancrofti: a doubleblind, randomised placebo-controlled trial. Lancet 365, 2116-2121.

Turelli, M. \& Hoffmann, A. A. (1991). Rapid spread of an inherited incompatibility factor in California Drosophila. Nature 353, 440-442.

Vavre, F., Fleury, F., Lepetit, D., Fouillet, P. \& Bouletreau, M. (1999). Phylogenetic evidence for horizontal transmission of Wolbachia in host-parasitoid associations. Mol Biol Evol 16, 1711-1723.

Wang, D. Y., Kumar, S. \& Hedges, S. B. (1999). Divergence time estimates for the early history of animal phyla and the origin of plants, animals and fungi. Proc Biol Sci 266, 163-171.

Wu, M., Sun, L. V., Vamathevan, J., Riegler, M., Deboy, R., Brownlie, J. C., McGraw, E. A., Martin, W., Esser, C. \& other authors (2004). Phylogenomics of the reproductive parasite Wolbachia pipientis wMel: a streamlined genome overrun by mobile genetic elements. PLoS Biol 2, E69. 